Pensamiento Crítico Vol.17 N² 2, pp. 155-160

\title{
Un modelo de demanda de dinero intertemporal con costo de transacciones
}

\author{
Richard Roca Garay ${ }^{1}$
}

\section{RESUMEN}

El presente trabajo expone un modelo de demanda de dinero intertemporal en la que los agentes toman en cuenta que el dinero reduce los costos de transacción de comprar bienes de consumo.

Palabras clave: Demanda de dinero, modelo intertemporal, costo de transacciones, teoría monetaria.

\begin{abstract}
This paper presents a model of intertemporal money demand in which agents take into account that the money reduces transaction costs of buying consumer goods.

Keywords: Money demand, inter temporal model, transactions cost, monetary theory.
\end{abstract}

1 Profesor de Economía de la Universidad Nacional Mayor de San Marcos. Correo electrónico: rhroca@ yahoo.com. Página web: http://economia.unmsm.edu.pe/prof/rroca 


\section{Introducción}

Los modelos intertemporales más conocidos han enfatizado en diversas bondades del dinero. El modelo de Generaciones yuxtapuestas de Paul Samuelson (1958) pone de relieve la función del dinero como medio de ahorro de largo plazo. Los Modelos MIU (Money in Utility function) se basan en Don Patinkin (1965) que incluye el dinero en una función de utilidad y Miguel Sidrauski (1967) considera al dinero como algo que influye positivamente en el bienestar de los agentes económicos. Bennett McCallum (1989) desarrolla el modelo de Shopping Time considerando al dinero como un medio de pago que reduce el tiempo necesario para comprar y por lo tanto deja más tiempo para el descanso lo cual mejora el bienestar. El modelo CIA enfatiza que para comprar bienes solo se puede hacer con dinero, no con otros bienes como indicara Clower (1967), la versión moderna es desarrollada por Robert Lucas (1982). Los mencionados modelos son presentados en las notas de clase de Richard Roca (2010). El modelo que desarrollamos es una variante de los Modelos Shopping Time considerando que los costos de transacción implican uso de recursos el cual se reduce cuando se cuenta con dinero y no solo su efecto a través del tiempo que se ahorra para el descanso.

\section{El modelo}

Consideremos un individuo hipotético en el periodo $t$ que busca maximizar el valor presente del bienestar del resto de su vida el cual depende en cada periodo del nivel de consumo $(c)$ y descanso $(\Theta)$. Suponiendo aditividad y separabilidad en la función de utilidad:

$$
U_{t}=\sum_{j=0}^{\infty} \beta^{j} u\left(c_{t+j}, \Theta_{t+j}\right)
$$

Donde $0<\beta<1$ es el factor de descuento intertemporal lo que implica que los individuos son impacientes.

Se supone utilidad marginal positiva pero decreciente del consumo y del ocio:

$$
u_{c}>0>u_{c c}, u_{\Theta}>0>u_{\Theta \Theta}
$$




\section{Richard Roca Garay}

Como en el modelo Shopping Time supongamos que a mayor nivel de consumo se requiere más tiempo para hacer las compras por lo que se tiene menos tiempo para el descanso. Por otro lado, a mayor cantidad real de dinero se puede hacer más rápido las compras quedando más tiempo para el descanso. La función del ocio en t:

$$
\Theta_{t}=\Theta\left(c_{t}, m_{t}\right)
$$

Además, se supondrá efectos marginales decrecientes. A mayor nivel de consumo se reduce el ocio pero se reduce cada vez menos, y a mayor tenencia real de dinero se incrementa el tiempo para el descanso pero se incrementa cada vez menos.

$$
\Theta_{c c}>0>\Theta_{m m}
$$

(2) en (1):

$$
U_{t}=u\left(c_{t}, \Theta\left(c_{t}, m_{t}\right)\right)+\beta u\left(c_{t+1}, \Theta\left(c_{t+1}, m_{t+1}\right)\right)+\beta^{2} u\left(c_{t+2}, \Theta\left(c_{t+2}, m_{t+2}\right)\right)+\ldots
$$

Cada periodo el agente recibe un ingreso real, $y$, e intereses por préstamos anteriores, que lo puede usar para comprar bienes de consumo, $c$, prestar comprando bonos, $B$, que vencen en un periodo y pagan intereses a la tasa nominal, $i$, o aumentar la posesión de dinero $M$. Adicionalmente consideramos que comprar bienes y servicios implica incurrir en costos de transacción $(T)$ por lo que en el periodo $t$ enfrenta la Restricción Presupuestaria:

$$
\mathrm{i}_{\mathrm{t}-1} \mathrm{~B}_{\mathrm{t}-1}+\mathrm{P}_{\mathrm{t}} \mathrm{y}_{\mathrm{t}}=\mathrm{P}_{\mathrm{t}} \mathrm{c}_{\mathrm{t}}+\mathrm{P}_{\mathrm{t}} \mathrm{T}_{\mathrm{t}}+\mathrm{M}_{\mathrm{t}}-\mathrm{M}_{\mathrm{t}-1}+\mathrm{B}_{\mathrm{t}}-\mathrm{B}_{\mathrm{t}-1}
$$

Supondremos que el costo de transacción real de comprar bienes de consumo depende directamente del nivel de consumo e inversamente de los saldos reales pues el dinero permite comprar con mayor rapidez:

$$
\underset{t}{T}=\underset{+}{\left(c_{t}, m_{t}\right)}
$$




\section{Pensamiento Crítico Vol. $17 \mathrm{~N}^{\circ} 2$}

Además, se supondrá efectos marginales decrecientes. A mayor nivel de consumo se incrementa los costos de transacción pero cada vez menos y a mayor nivel de saldos reales se reduce los costos de transacción pero se reduce cada vez menos.

$$
T_{c c}<0<T_{m m}
$$

(5) en (4) y reordenando

$$
\begin{gathered}
i_{t-1} B_{t-1}+P_{t} y_{t}=P_{t} c_{t}+P_{t} T_{t}\left(c_{t}, m_{t}\right)+M_{t}-M_{t-1}+B_{t}-B_{t-1} \\
B_{t-1}=\frac{P_{t}\left[c_{t}-y_{t}+T_{t}\left(c_{t}, m_{t}\right)\right]+M_{t}-M_{t-1}+B_{t}}{\left(1+i_{t-1}\right)}
\end{gathered}
$$

En los demás periodos se repite la restricción:

$$
\begin{aligned}
& B_{t}=\frac{P_{t+1} c_{t+1} y_{t+1}+T_{t+1}\left(c_{t+1}, m_{t+1}\right)+M_{t+1} M_{t}+B_{t+1}}{\left(1+i_{t}\right)} \\
& B_{t+1}=\frac{P_{t+2}\left[c_{t+2}-y_{t+2}+T_{t+2}\left(c_{t+2}, m_{t+2}\right)\right]+M_{t+2}-M_{t+1}+B_{t+2}}{\left(1+i_{t+1}\right)}
\end{aligned}
$$

Reemplazando una en otra sucesivamente se obtiene la Restricción Presupuestaria Intertemporal: 


\section{Richard Roca Garay}

$$
\begin{gathered}
B_{t-1}=\frac{P_{t}\left[c_{t}-y_{t}+T_{t}\left(c_{t}, m_{t}\right)\right]+M_{t}-M_{t-1}}{\left(1+i_{t-1}\right)}+\frac{P_{t+1}\left[c_{t+1}-y_{t+1}+T_{t+1}\left(c_{t+1}, m_{t+1}\right)\right]+M_{t+1}-M_{t}}{\left(1+i_{t-1}\right)\left(1+i_{t}\right)}+ \\
+\frac{P_{t+2}\left[c_{t+2}-y_{t+2}+T_{t+2}\left(c_{t+2}, m_{t+2}\right)\right]+M_{t+2}-M_{t+1}}{\left(1+i_{t-1}\right)\left(1+i_{t}\right)\left(1+i_{t+1}\right)}+\ldots
\end{gathered}
$$

por lo que la función lagrangeana:

$$
\begin{aligned}
& L_{t}=u\left(c_{t}, \Theta\left(c_{t}, m_{t}\right)\right)+\beta u\left(c_{t+1}, \Theta\left(c_{t+1}, m_{t+1}\right)\right)+\beta^{2} u\left(c_{t+2}, \Theta\left(c_{t+2}, m_{t+2}\right)\right)+\ldots \\
& \lambda_{t}\left\{\begin{array}{r}
P_{t}\left[c_{t}-y_{t}+T_{t}\left(c_{t}, m_{t}\right)\right]+M_{t}-M_{t-1} \\
+-B_{t-1}+\frac{P_{t+1}\left[c_{t+1}-y_{t+1}+T_{t+1}\left(c_{t+1}, m_{t+1}\right)\right]+M_{t+1}-M}{+i_{t-1}}+\frac{\left(1+i_{t-1}\right)\left(1+i_{t}\right)}{\left.1+i_{t}\right)}
\end{array}\right.
\end{aligned}
$$

Dado que en el periodo $t$ el individuo debe decidir cuánto consumir, con cuánto dinero terminar el periodo, y por diferencia, eso daría el saldo acreedor con el que se termina el periodo $t$, las condiciones de primer orden son:

$$
\begin{aligned}
& \frac{\partial L_{t}}{\partial C_{t}}=0: \quad u_{c_{t}}+u_{\Theta_{t}} \Theta_{c_{t}}+\lambda_{t}\left\{\frac{P_{t}\left[1+T_{c_{t}}\right]}{1+i_{t-1}}\right\}=0 \\
& \frac{\partial L_{t}}{\partial M_{t}}=0: \quad u_{\Theta_{t}} \Theta_{m_{t}} \frac{1}{P_{t}}+\lambda_{t}\left\{\frac{T_{m_{t}}+1}{1+i_{t-1}}-\frac{1}{\left(1+i_{t-1}\right)\left(1+i_{t}\right)}\right\}=0
\end{aligned}
$$

Combinando las anteriores:

$$
u_{\Theta_{t}} \Theta_{m_{t}}\left[1+T_{c_{t}}\right]=\left(u_{c_{t}}+u_{\Theta_{t}} \Theta_{c_{t}}\left[T_{m_{t}}+\frac{i_{t}}{\left(1+i_{t}\right)}\right]\right.
$$




\section{Pensamiento Crítico Vol. $17 \mathrm{~N}^{\circ} 2$}

Esta es la condición de optimalidad del individuo la cual involucra a las variables: $c_{t}, m_{t}, i_{t}$. De donde, a pesar de lo engorroso, se obtiene una función de demanda real de dinero óptima de un individuo:

$$
m_{t}^{d}=L\left(c_{t}, i_{t}\right)
$$

\section{Referencias bibliográficas}

Clower, R. (1967). A Reconsiderations of the Microfundations of Monetary Theory. En Western Economic Journal.

McCallum, B. (1989). Monetary Economics. Theory and Policy. McMillan.

Lucas, R. (1982). "Interest Rates and Currency Prices in a Two Country Model", Journal of Monetary Economics.

Patinkin, D. (1965). Money, Interest and Prices, New York: Haper \& Row.

Roca, R. (2010). Teoría y política monetaria. Lima: Universidad Nacional Mayor de San Marcos.

Samuelson, P. A. (1958). "An Exact Model of Loan consumption and Interest Rate with or without the social contrivance of Money". Journal of Political Economy.

Sidrauski (1967) "Rational Choice and Patterns of Growth in a Monetary Economy". En American Economic Review. 